\title{
SARS-CoV-2 vaccination in gynecologic oncology
}

\author{
Zachary Alholm ${ }^{1, *}$, Daniel Spinosa ${ }^{2}$, Rebecca A. Previs ${ }^{3}$, Stephanie L. Shuey ${ }^{4}$, David M. O'Malley ${ }^{5}$, Leslie Randall ${ }^{6}$, \\ Brian Slomovitz ${ }^{7}$, Bradley ]. Monk ${ }^{8}$ \\ ${ }^{1}$ Department of Obstetrics and Cynecology, University of Arizona-Phoenix, Phoenix, AZ 85004, USA \\ ${ }^{2}$ Department of Obstetrics and Gynecology, Duke University Health System, Durham, NC 27710, USA \\ ${ }^{3}$ Department of Obstetrics and Cynecology, Division of Cynecologic Oncology, Duke Cancer Institute, Duke University Medical Center, Durham, NC 27710, \\ USA \\ ${ }^{4}$ Department of Pharmacy, Duke University Medical Center, Durham, NC 27710, USA \\ ${ }^{5}$ Division of Cynecology Oncology, The Ohio State University and the James Comprehensive Cancer Center, Columbus, OH 43210, USA \\ ${ }^{6}$ Division of Cynecologic Oncology, Massey Cancer Center, Virginia Commonwealth University, Richmond, VA 23284, USA \\ ${ }^{7}$ Division of Cynecology Oncology, Broward Health, Florida International University, Fort Lauderdale, FL 33199, USA \\ ${ }^{8}$ Arizona Oncology (US Oncology Network), University of Arizona, Creighton University, Phoenix, AZ 85721, USA \\ *Correspondence: zalholm@gmail.com (Zachary Alholm)
}

DOI:10.31083/j.ejgo.2021.02.2491

This is an open access article under the CC BY 4.0 license (https://creativecommons.org/licenses/by/4.0/).

Submitted: 13 April 2021 Accepted: 15 April 2021 Published: 15 April 2021

Severe Acute Respiratory Syndrome Coronavirus 2 (SARS-CoV-2) is a novel human respiratory virus with the first reported cluster of cases documented in Wuhan, China on 31 December 2019. After its initial spread, it was officially declared a pandemic by the World Health Organization on 11 March 2020, and is the fifth documented pandemic since the 1918 Influenza. As of 13 April 2021, there have been over 137 million cases worldwide with 31 million in the United States (mortality rates of $2.2 \%$ and $1.8 \%$, respectively) and continued viral spread expected in the coming months [1]. One of the defining features of the virus is its unpredictable course, with some people living as asymptomatic vectors while others suffer severe illness or even death. An emerging body of evidence suggests that people with co-morbidities are more susceptible to severe manifestations of the virus, with oncology patients being particularly vulnerable. Cancer patients have a higher SARS-CoV-2-associated mortality rate when compared to non-cancer patients (20\% to $11 \%$ [2]). With the rising prevalence of SARS-CoV-2 infections, there is a pressing need for interventions to protect oncology patients, including gynecologic oncology patients.

Recently, two mRNA SARS-CoV-2 vaccines were granted Emergency Use Authorization (EUA) by the Food and Drug Administration (FDA) [3] based on data reported in two clinical trials that demonstrated 94-95\% efficacy for the prevention of SARS-CoV-2 infection in the non-cancer population [4, 5]. Expedited distribution of these vaccines is currently underway in the United States with administration according to Centers for Disease Control (CDC)/Advisory Committee on Immunization Practices (ACIP) prioritization of recipients delegated to individual states [6]. Despite being listed as high priority (CDC Phase Ib), cancer patients were excluded from these pivotal phase III safety and efficacy trials. This clearly complicates decisions about if and when to vaccinate oncology patients. As such, oncology patients receiving the vaccine will require additional supporting evidence. This may include studies from other populations included in the trials, evidence from other vaccines, and a risk versus benefit analysis weighing the risks of administering the vaccine to an understudied population against the known risk of the disease in a vulnerable population.

The mRNA vaccines utilize a novel mechanism of action, which is important to understand as we consider the safety profile in oncology patients. Both SARS-CoV-2 vaccines approved in the U.S. are lipid nanoparticle-formulated, nucleoside-modified mRNA vaccines encoding the prefusion spike glycoprotein of SARS-CoV-2. The vaccine contains mRNA inside a lipid capsule, which prevents its premature breakdown and allows for uptake into antigen presenting cells. After cellular uptake, the mRNA acts as instruction for the cell to produce the spike protein specific to SARS-CoV2. Once the spike proteins are made, the mRNA is quickly degraded in the cytoplasm. The spike proteins are then expressed on the cell surface and provoke an immune response, allowing for production of antibodies and activating helper T-cells [7]. While the study of memory B-cell response requires more longitudinal data, studies show a robust helper $\mathrm{T}$-cell response after receiving the two-dose vaccine series $[4,5,8]$.

This mechanism of action is distinct from other recommended vaccines, especially those which are live-attenuated and generally contraindicated in oncology patients due to their potential to cause disease in an immunocompromised host. No live virions are present in either of the current vac- 
cines, so there is no risk of contracting SARS-CoV-2 from the vaccine itself. Further, the safety profile in the published clinical trials $[4,5]$ is similar to other viral vaccines, including in subpopulations with other stable coexisting conditions such as human immunodeficiency virus (HIV), hepatitis B virus, or hepatitis $\mathrm{C}$ virus (though current HIV safety data remains limited). Commonly reported adverse events include local reactions such as pain, erythema, and swelling at the injection site and regional lymphadenopathy as well as systemic symptoms such as fever, headache, fatigue, myalgia, arthralgia, and chills $[4,5]$. In clinical trials, hypersensitivityrelated adverse events were observed in $0.63 \%$ of participants who received the Pfizer-BioNTech COVID-19 vaccine and $1.5 \%$ of participants who received the Moderna COVID-19 vaccine, compared to $0.51 \%$ and $1.1 \%$, respectively, in the placebo groups. Anaphylaxis following vaccination was not observed in the Pfizer-BioNTech or Moderna COVID-19 vaccines clinical trials. Though the vaccine safety profile in immunocompromised populations is unknown, no unique adverse effects have been noted after receiving other available inactivated vaccines [9] and the Center for Disease Control recommends immunocompromised individuals may receive COVID-19 vaccination if they have no known contraindications [6].

One concern of mRNA vaccine administration in an immunocompromised oncology population is efficacy. The primary concern is that, due to immune dysregulation or suppression, the vaccine would not generate the requisite immune response in oncology patients to provide lasting protection. While the validity of this concern is unknowable at this time, there is reason to be optimistic about vaccine effectiveness. Overall, the vaccines were $94 \%$ and $95 \%$ effective $[4,5]$. Importantly, the mRNA vaccines were shown to be effective in high risk populations including stable HIV, hepatitis $B$, and hepatitis $C$ patients $[4,5]$.

While mRNA vaccines were the first to be FDA approved, other types of vaccines have recently been approved for SARS-CoV-2 vaccination. The adenovirus 26 (Ad26) recombinant vector-based vaccine is an inactivated viral-vector vaccine sponsored by Johnson and Johnson and received emergency authorization from the FDA on February 27th, 2021 for vaccination use against the SARS-CoV-2 [10]. Ad26 has yet to be tested in oncology patients, is viral-vector based, and as of 12 April 2021 was put on pause by the CDC and FDA over blood clot concerns [11]. Given the availability of mRNA vaccines and these recent concerns, we currently do not recommend the Ad26 vaccine for our gynecology oncology patients.

There are other notable examples of inactivated vaccine use in oncology patients. Specifically, it is recommended that patients with cancer receive influenza and pneumococcal vaccines. The rationale is that the risk of developing serious complications from these infections is markedly increased in cancer patients, so even a blunted immune response following vaccination may confer clinically meaningful benefits.
Other inactivated vaccines such as zoster, tetanus, diphtheria, and acellular pertussis (Tdap), meningococcal, human papillomavirus (HPV), Haemophilus influenzae type b (Hib), and hepatitis A and B should also be considered if indicated based on patient age and/or comorbidities. It is recommended that these vaccines be administered at least 14 days prior to initiation of chemotherapy or 3 months after completion to ensure a robust immune response [9].

Available data about the current vaccines and precedent from other vaccines points towards SARS-CoV-2 mRNA vaccines likely being safe and effective (at least to some degree) against a virus that is reported to have excess morbidity and mortality among oncology patients [2]. Based on currently available data, we believe the anticipated benefits of vaccination outweigh the risks. In line with January 22nd, 2021 COVID-19 NCCN guidelines [12], we favor offering the mRNA vaccine to any interested gynecologic oncology patients, though providers should be transparent about the relative lack of data supporting that recommendation. These recommendations include prioritization of gynecologic oncology patients for vaccination (CDC priority group $1 \mathrm{~b} / \mathrm{c}$ ), immunization for patients receiving active treatment, immunosuppressive therapy such as chemotherapy and radiation (with the understanding there is limited safety and efficacy data), and caregivers/household/close contacts should be immunized when possible. Current vaccine prioritization includes patients with active cancer, those planning to start treatment, and those immediately ( $<6$ months) posttreatment; however, patients without active cancer who are receiving hormonal therapy only would have lower prioritization. There is currently no role for revaccination or booster doses of mRNA COVID-19 vaccines after immunocompetence is restored. Antibody testing may play a future role in revaccination efforts but should not be used to screen current patients for vaccine eligibility or modify the two-dose regimens that have been studied. As with non-oncology patients, history of the SARS-CoV-2 infection is not a contraindication to vaccination, given that reinfection is possible.

Most importantly, we continue to emphasize the need for distancing, masking, and washing hands, all of which are strongly supported by available evidence and without risk to oncology patients. After vaccination, it is important to continue to follow these precautions as patients may still be at risk for infection and/or spread the virus to others.

\section{Author contributions}

ZA, DS and BJM originated the clinical commentary. RAP, DMO, LR, BS and BJM provided gynecologic oncology guidance. SLS contributed pharmacologic expertise. All authors contributed to editorial changes in the manuscript. All authors read and approved the final manuscript.

\section{Ethics approval and consent to participate}

Not applicable. 


\section{Acknowledgment}

We'd like to express our gratitude to all of those who helped in the writing of this manuscript.

\section{Funding}

This research received no external funding.

\section{Conflict of interest}

ZA reports no conflicts. DS has an equity holding in Invitae via his wife's employment with the company. RP has served on Myriad advisory boards and has received research funding. SS has served on an Eisai advisory board. DO reports personal fees from consulting and/or advisory board member from AstraZeneca, Tesaro/GSK, Immunogen, Ambry, Janssen/J\&J, Abbvie, Regeneron, Amgen, Novocure, Genentech/Roche, GOG Foundation, Iovance Biotherapeutics, Inc, Myriad Genetics, Eisai, Agenus, Tarveda, Merck, SeaGen, Novartis, Mersana, Clovis, Rubis, Elevar. Research Funding (all funding to institution): AstraZeneca, Tesaro/GSK, Immunogen, Janssen/J\&J, Abbvie, Regeneron, Amgen, Novocure, Genentech/Roche, VentiRx Array Biopharma EMD Serono, Ergomed, Ajinomoto Inc., Ludwig Cancer Research Stemcentrx, Inc, CERULEAN PHARMA, GOG Foundation, NCI, Bristol-Myers Squibb Co, Serono Inc, TRACON Pharmaceuticals, Yale University, New Mexico Cancer Care Alliance, INC Research, Inc, inVentiv Health Clinical, Iovance Biotherapeutics, Inc, PRA Intl, Eisai, Agenus, Merck, GenMab, SeaGen, personal fees from Mersana, Clovis. LR reports personal fees from consulting and/or advisory board member from Agenus, GSK, AstraZeneca, Myriad, Genentech/Roche, GOG Foundation, Clovis, Rubius, BluPrint Oncology, EMD Serono/GSK, Mersana, Merck, and Akeso Biopharma. BS reports personal fees from Consulting and/or advisory board member from AstraZeneca, Tesaro/GSK, ImmunogenAbbvie, Novocure, Genentech/Roche, GOG Foundation, Myriad Genetics, Eisai, Agenus, Merck, SeaGen, Novartis, Clovis, Rubis. BM reports personal fees from consulting, speaking and/or advisory board member from AstraZeneca, Tesaro/GSK, Genentech/Roche, Clovis, GOG Foundation, Merck, Myriad Genetics, Eisai, and consulting fees from Agenus, SeaGen/Genmab, Rubis, Sorento, Iovance, Mer- sana, Novocure.

\section{References}

[1] Dong E, Du H, Gardner L. An interactive web-based dashboard to track COVID-19 in real time. Lancet Infectious Diseases. 2020; 20: $533-534$

[2] Hachem RY, Datoguia T, Siddiqui B, Cruz AF, Mori N, Fakhreddine S, et al. 372. Comparing the outcome of COVID-19 in cancer and non-cancer patients: an international multicenter study. Open Forum Infectious Diseases. 2020; 7: S256.

[3] United States Food and Drug Administration (FDA) Press Release. FDA Takes Additional Action in Fight Against COVID-19 By Issuing Emergency Use Authorization for Second COVID-19 Vaccine. 2020. Available at: https://www.fda.gov/news-events/pres s-announcements/fda-takes-additional-action-fight-against-C OVID-19-issuing-emergency-use-authorization-second-covid (Accessed: 18 January 2021).

[4] Baden LR, El Sahly HM, Essink B, Kotloff K, Frey S, Novak R, et al. Efficacy and safety of the mRNA-1273 SARS-CoV-2 vaccine. New England Journal of Medicine. 2021; 384: 403-416.

[5] Polack FP, Thomas SJ, Kitchin N, Absalon J, Gurtman A, Lockhart $\mathrm{S}$, et al. Safety and efficacy of the BNT162b2 mRNA COVID-19 vaccine. New England Journal of Medicine. 2020; 383: 2603-2615.

[6] Centers for Disease Control and Prevention (CDC). Interim clinical considerations for use of mRNA COVID-19 vaccines currently authorized in the United States. 2021. Available at: https://www.cdc.gov/vaccines/COVID-19/info-by-product /clinical-considerations.html (Accessed: 24 Janurary 2021).

[7] Poland GA, Ovsyannikova IG, Kennedy RB. SARS-CoV-2 immunity: review and applications to phase 3 vaccine candidates. Lancet. 2020; 396: 1595-1606.

[8] Widge AT, Rouphael NG, Jackson LA, Anderson EJ, Roberts PC, Makhene M, et al. Durability of responses after SARS-CoV2 mRNA-1273 vaccination. New England Journal of Medicine. 2021; 384: 80-82.

[9] Rubin LG, Levin MJ, Ljungman P, Davies EG, Avery R, Tomblyn M, et al. 2013 IDSA clinical practice guideline for vaccination of the immunocompromised host. Clinical Infectious Diseases. 2014; 58: e44-e100.

[10] Johnson \& Johnson. Johnson \& Johnson COVID-19 Vaccine Authorized by U.S. FDA for Emergency Use-First Single-Shot Vaccine in Fight Against Global Pandemic. 2021. Available at: https://www.jnj.com/johnson-johnson-COVID-19-vaccine-aut horized-by-u-s-fda-for-emergency-usefirst-single-shot-vaccine -in-fight-against-global-pandemic (Accessed: 05 April 2021).

[11] Johnson \& Johnson. Johnson \& Johnson Statement on COVID-19 Vaccine. 2021. Available at: https://www.jnj.com/johnson-johns on-statement-on-COVID-19-vaccine (Accessed: 13 April 2021).

[12] National Comprehensive Cancer Network. Cancer and COVID-19 Vaccination (Version 1.0.2021). 2021. Available at: https://www.nccn.org/COVID-19/pdf/COVID-19_Vaccinatio n_Guidance_V1.0.pdf (Accessed: 24 January 2021). 